\title{
ADDENDUM
}

\section{Not Just How Much You Know: Interactional Effect of Cultural Knowledge and Metacognition on Greativity in a Global Context-ADDENDUM}

\author{
Roy Y. J. Ghua ${ }^{1}$ and Kok Yee $\mathbf{N g}^{2}$ \\ ${ }^{1}$ Singapore Management University, Singapore, and ${ }^{2}$ Nanyang Technological University, Singapore
}

doi:https://doi.org/10.1017/mor.2016.32, Published by Gambridge University Press, 1 December 2016.

KEYWORDS creativity, cultural intelligence, cultural metacognition, knowledge, teams, addendum

In view of MOR's recent changes to editorial policies (Lewin et al., 2016), this addendum is created to provide greater data transparency and richer discussions of our findings and their implications.

Table 2 is revised to include the exact p-values for each effect.

\section{Discussion of Control Variables}

(a) Network properties: Diversity and size. Models 2 suggests that individuals whose social networks are culturally diverse are more creative. This is consistent with current research, which found that people with culturally diverse networks are better able to creatively solve problems that requires knowledge from multiple cultures (Chua, 2015). Network size, however, did not have any significant effect on creativity although one might expect that a larger network could provide more knowledge resources for individuals to draw on during creative work. One explanation could be that for creativity, it is not so much the amount of cognitive resources that one is exposed to that matters but the diversity of these cognitive resources. Thus, a large network that provides a large amount of redundant resources is not 
Table 2. Random effects regression results $(\mathrm{N}=89)$

Dependent variable $=$ Creativity $\quad$ Model 1

Model 1

Model 2

Model 3

Model 4

Model 5

\section{Key predictors}

Cultural Knowledge

Cultural Knowledge-Squared

Cultural Metacognition

Cultural Knowledge x Metacognition

Cultural Knowledge-Squared x

\section{Metacognition}

\section{Control variables}

Network cultural diversity

Network size

Age

Gender $($ Male $=1$, Female $=0$ )

Number of languages known

Experience interacting with foreigners

Number of countries lived ( $>6 \mathrm{mths}$ )

Number of countries visited

Extraversion

Agreeableness

Conscientiousness

Emotional stability

Openness

Team size

Within R-Square

Between R-Square

Overall R-Square

Overall R-Square change

$$
0.29[0.03]^{*}(0.13)
$$$$
-
$$$$
-
$$

$-$

$0.80[0.13](0.53)$ $0.01[0.34](0.01)$

$-0.06[0.28](0.05)$ $0.01[0.98](0.23)$

$0.00[1.00](0.10)$

$-0.18[0.44](0.24)$

$0.01[0.94](0.11)$ $0.03[0.04]^{*}(0.01)$ 0.08 [0.62] $(0.16)$

$0.23[0.40](0.27)$

$-0.40[0.04]^{*}(0.19)$

$-0.10[0.56](0.16)$

$-0.53[0.04]^{*}(0.26)$

$0.15[0.36](0.16)$

0.047

0.638

0.263

\begin{abstract}
$1.00[0.04]^{*}(0.48)$ $0.01[0.21](0.01)$

$-0.06[0.24](0.05)$

$-0.06[0.78](0.21)$

$-0.02[0.82](0.10)$

$-0.29[0.19](0.22)$

$-0.02[0.85](0.10)$

$0.05[0.01]^{* *}(0.01)$

$-0.10[0.53](0.16)$

0.15 [0.54] (0.25)

$-0.35[0.04]^{*}(0.17)$

$-0.02[0.87](0.15)$

-0.45 [0.05] $(0.24)^{*}$

0.12 [0.42] (0.15)

0.110

0.778

0.394
\end{abstract}

$-0.08[0.61](0.16)$

$-0.40[0.00]^{* *}(0.13)$ $0.41[0.00]^{* *}(0.13)$ $-$

$-$
$-0.34[0.054]+(0.18)$

-0.19 [0.48] $(0.27)$

$0.24[0.08]+(0.14)$

0.00 [0.98] (0.21)

$0.30[0.00]^{* *}(0.09)$
$-0.31[0.013]^{*}(0.13)$

$-0.16[0.44](0.20)$

$0.06[0.61](0.11)$

$0.11[0.51](0.17)$

$0.28[0.00]^{* *}(0.07)$

Notes: Numbers in square brackets $\square$ are p-values; numbers in parenthesis 0 are standard errors. $+\mathrm{p}<0.10$; $^{*} \mathrm{p}<0.05,{ }^{* *} \mathrm{p}<0.01$

$0.74[0.94]+(0.44)$

$0.00[0.96](0.01)$

$-0.06[0.13](0.04)$

-0.14 [0.47] (0.19)

$-0.02[0.86](0.09)$

$-0.27[0.16](0.20)$

$0.00[0.96](0.09)$

$0.03[0.01]^{* *}(0.01)$

$-0.09[0.54](0.14)$

$-0.01[0.78](0.24)$

$-0.28[0.07]+(0.16)$

$-0.09[0.49](0.14)$

$-0.22[0.31](0.22)$

$0.12[0.38](0.13)$

0.249

0.817

0.535

0.070
0.194

0.557

0.351 
as helpful for creativity than a small network that provides a smaller but more diverse range of resources.

(b) Demographics: Age and gender. Age did not have any significant effect on individuals' creativity. Because older individuals might have more knowledge resources and experiences to draw on for creative work, it is useful to control for this variable. The null effect might be a result of the limited range in the age variable given that participants are of similar ages (22.19 years; $\mathrm{SD}=2.06$ years). Research on gender and creativity generally suggests that men and women do not differ on creative abilities (see Baer \& Kaufman, 2008 for review). Thus, we do not expect significant differences between gender on creativity.

(c) Language ability. The number of language known does not have any significant effect on creativity. Research on multilingualism on creativity has to date yielded mixed results (Kharkhurin, \& Wei, 2015; Leikin \& Tovli, 2014; Paap \& Greenberg, 2013; Paap, 2014). Specifically, Kharkhurin and Wei (2015: 1) argued that codeswitching, 'the alternation and mixing of different languages in the same episode of speech production', a process inherent in bilingual activities, promotes divergent thinking. However, Paap and Greeberg (2013) argued that there is no coherent evidence for multilingual advantage on cognitive processes that could aid creativity in part because prior studies tend to focus on a narrow category of tasks. Our null finding appears consistent with the latter claim.

(d) Cultural experiences. Our finding that the number of countries participants visited has a positive effect on creativity is consistent with Godart, Maddux, Shipilov, and Galinsky (2015) finding that breadth of foreign exposure (i.e., number of countries visited) is positively associated with creative performance. However, it is interesting to note the null effects for the number of countries lived and the amount of experience interacting with foreigners on creativity. These findings are inconsistent with arguments that living overseas and mere exposure to foreign cultures can promote creativity (Leung, Maddux, Galinsky, \& Chiu, 2008; Maddux \& Galinsky, 2009). Taking together our current findings and those of prior research, it appears that the relationship between different types of cultural exposure and creativity may be more complex than otherwise thought. Future research should examine more nuanced theories about when and how different forms of multicultural experiences might influence creative thinking and performance.

(e) Personality. The key significant effect here is that openness appears to have a negative impact on creativity. We have discussed this unexpected finding in the main paper. Interestingly, there also appeared to be a negative effect of conscientiousness on creativity 
(see Model 1). One speculation is that conscientiousness might be more relevant for structured and well-defined tasks where putting in extra effort would yield positive results. Given the unstructured nature of creative work, high conscientiousness may have a negative effect when it leads one to keep trying conventional methods of solving problems without thinking outside the box. Future research might further explore the relationship between conscientiousness and creativity. As for extraversion, emotional stability, and agreeableness, there are no compelling theoretical reasons to expect any effect on creativity. These variables are included for completeness so that all Big-Five personality variables are controlled for.

(f) Team size. Team size did not have any significant effect on individuals' creativity. Although it is plausible that larger teams might confer greater knowledge resources to the individual members thus increasing their creativity, team size in the current study might be range-limited (5-7 persons per team). Additionally, as we argued above in (a), it is not the amount but rather the diversity of knowledge resources that matters more.

\section{Discussion of Effect Size and Implications}

Our hypothesized relationships $(\mathrm{H} 1$ and $\mathrm{H} 2)$ accounted for a total of $27 \%$ of variance in creativity. These results show that while cultural knowledge and cultural metacognition do explain differences in creativity in intercultural tasks, there remains a substantial amount of unexplained variance. This is not surprising, given that our model had aimed to address the paradoxical effects of individuals' domain-relevant knowledge on creativity. As Cortina and Landis (2009) pointed out, research questions that examine counter-intuitive relationships should be expected to have smaller magnitudes. This does not, however, mean that the results are not practically important. In our case, the findings highlight a practically useful advice that having more cultural knowledge may not be increasingly beneficial for creativity as conventional wisdom would suggest and that there is a potential downside. This downside, however, can be mitigated by honing one's cultural metacognition.

Still, our current model has not considered important contextual factors that will affect individual creativity, as suggested by the componential model (Amabile, 1983) and interactionist perspective (e.g., Woodman, Sawyer, \& Griffin, 1993) of creativity. In the context of our study, team context should play an important role in affecting individuals' creativity. Recent studies on individual creativity in teams have found that factors such as team learning goal orientation (Gong, Kim, Lee, \& Zhu, 2013) and team learning behaviors (Hirst, van Knippenberg, \& Zhou, 2009) positively affect team members' creativity. We suggest that future research could examine whether team goal orientation and learning behaviors 
will exert cross-level moderating effects to mitigate the detrimental effect of excessive cultural knowledge. For instance, individuals situated in teams that actively seek different perspectives from members and reflect on decision-making processes may be less subject to cognitive limitations posed by their existing domain-relevant knowledge. By incorporating factors that assess the context in which creativity takes place, future research can offer findings with greater practical significance to organizations that require creativity in inter-cultural tasks.

In addition, as suggested by Aguinis and colleagues (2010), future research could include qualitative methods to better assess the practical significance of this research inquiry. For instance, future research could use narrative inquiry to understand how global leaders use their cultural knowledge and cultural metacognition to create new ideas and products; when they encounter cognitive overload and entrenchment, and how they overcome these cognitive limitations.

\section{REFERENGES}

Aguinis, H., Werner, S., Abbott, J. L., Angert, C., Park, J. H., \& Kohlhausen, D. 2010. Customercentric science: Reporting research results with rigor, relevance, and practical impact in mind. Organizational Research Methods, 13: 515-539.

Amabile, T. M. 1983. The social psychology of creativity: A componential conceptualization. Journal of Personality and Social Psychology, 45: 357-377.

Baer, J., \& Kaufman, J. C. 2008. Gender differences in creativity. The Journal of Creative Behavior, 42(2): 75-105.

Chua, R. Y. J. 2015. Innovating at cultural crossroads: How multicultural social networks promote ideas flow and creativity. Journal of Management. Online First, 3 September 2015.

Cortina, J. M., \& Landis, R. S. 2009. When small effect sizes tell a big story, and when large effect sizes don't. In C. E. Lance \& R. J. Vandenberg (Eds.), Statistical and methodological myths and urban legends: Doctrine, verity, and fable in the organizational and social sciences: 287-308. New York: Routledge.

Godart, F., Maddux, W. W., Shipilov, A. V., \& Galinsky, A. 2015. Fashion with a foreign flair: Professional experiences abroad facilitate the creative innovations of organizations. Academy of Management Journal, 58(1): 195-220.

Gong, Y., Kim, T., Lee, \& Zhu, J. 2013. A multilevel model of team goal orientation, information exchange and creativity. Academy of Management Journal, 56: 827-851.

Hirst, G., van Knippenberg, D., \& Zhou, J. 2009. A cross-level perspective on employee creativity: Goal orientation, team learning behavior, and individual creativity. Academy of Management Journal, 52: 280-293.

Kharkhurin, A. V., \& Wei, L. 2015. The role of code-switching in bilingual creativity. International Journal of Bilingual Education and Bilingualism, 18(2): 153-169.

Leikin, M., \& Tovli, E. 2014. Bilingualism and creativity in early childhood. Creativity Research Journal, 26(4): 411-417.

Leung, A. K., Maddux, W. W., Galinsky, A. D., \& Chiu, G. Y. 2008. Multicultural experience enhances creativity? The when and how. American Psychologist, 63(6): 169-181.

Lewin, A., Chiu, G.-Y., Fey, C. F., Levine, S. S., McDermott, G., Murmann, J. P., \& Tsang, E. W. K. 2016. The critique of empirical social science: New policies at Management and Organization Review. Management and Organization Review, 12(4): 649-665.

Maddux, M. W., \& Galinsky, A. D. 2009. Cultural borders and mental barriers: The relationship between living abroad and creativity. Journal of Personality and Social Psychology, 96: 1047-1061. 
Paap, K. R. 2014. The role of componential analysis, categorical hypothesising, replicability and confirmation bias in testing for bilingual advantages in executive functioning. Journal of Cognitive Psychology, 26(3): 242-255.

Paap, K. R., \& Greenberg, Z. I. 2013. There is no coherent evidence for a bilingual advantage in executive processing. Cognitive Psychology, 66(2): 232-258.

Woodman R., W., Sawyer, J. E., \& Griffin, R. W. 1993. Toward a theory of organizational creativity. Academy of Management Reviez, 18: 293-321. 